\title{
The strong deviation theorem for discrete-time and continuous-state nonhomogeneous Markov chains
}

Bei Wang ${ }^{*}$ and Zhiyan Shi

\section{${ }^{*}$ Correspondence:}

wangbei@ujs.edu.cn

Faculty of Science, Jiangsu

University, Zhenjiang, 212013, China

\begin{abstract}
In this paper, the notion of asymptotic average log-likelihood ratio, as a measure of the difference between the sequence of random variables and Markov chains, is introduced, and by constructing a nonnegative martingale, the strong deviation theorem for discrete-time and continuous-state nonhomogeneous Markov chains is established.
\end{abstract}

Keywords: discrete-time and continuous-state nonhomogeneous Markov chain; asymptotic average log-likelihood ratio; transition probability density; strong deviation theorem

\section{Introduction}

Let $(\Omega, \mathcal{F}, P)$ be the probability space, and let $\left\{X_{n}, n \geq 0\right\}$ be a sequence of continuous random variables taking values in $R$ and with the joint density function $f_{n}\left(X_{0}, X_{1} \cdots X_{n}\right), n=$ $1,2, \ldots$. Let $Q$ be another probability measure on $(\Omega, \mathcal{F})$, and $\left\{X_{n}, n \geq 0\right\}$ be an independent random sequence on the measure $Q$, with the joint density function $g_{n}\left(X_{0}, X_{1} \cdots X_{n}\right), n=$ $1,2, \ldots$

Let

$$
\begin{aligned}
& r_{n}(\omega)=\frac{g_{n}\left(X_{0}, X_{1} \cdots X_{n}\right)}{f_{n}\left(X_{0}, X_{1} \cdots X_{n}\right)}, \\
& r(\omega)=-\liminf _{n \rightarrow \infty} \frac{1}{n} \ln r_{n}(\omega) \quad(\ln 0=-\infty),
\end{aligned}
$$

where $\omega$ is a sample point. In statistical terms, $r_{n}(\omega)$ and $r(\omega)$ are called the likelihood ratio and the asymptotic average log-likelihood ratio, respectively [1]. Obviously, if $f_{n}\left(x_{0}, x_{1} \cdots x_{n}\right)=g_{n}\left(x_{0}, x_{1} \cdots x_{n}\right), n \geq 1$, then $r_{n}(\omega) \equiv 0$, a.s. So $r(\omega)$ can be used as a measure of deviation between $f_{n}\left(x_{0}, x_{1} \cdots x_{n}\right)$ and $g_{n}\left(x_{0}, x_{1} \cdots x_{n}\right)$ when $n$ tends to infinity. The smaller $r(\omega)$ is, the smaller the deviation is.

Definition 1 [2] Let $\left\{X_{n}, n \geq 0\right\}$ be a nonhomogeneous Markov chain with the initial distribution $u(x), x \in R$, and the transition probability density $p_{n}=p_{n}(x, y), x, y \in R, n \geq 1$. If

$$
P\left(X_{0} \in B\right)=\int_{B} u(x) d x, \quad P\left(X_{n+1} \in B \mid X_{n}=x\right)=\int_{B} p_{n}(x, y) d y,
$$

02013 Wang and Shi; licensee Springer. This is an Open Access article distributed under the terms of the Creative Commons Attribution License (http://creativecommons.org/licenses/by/2.0), which permits unrestricted use, distribution, and reproduction in any medium, provided the original work is properly cited. 
this Markov chain is called a discrete-time and continuous-state nonhomogeneous Markov chain.

Let $\left\{X_{n}, n \geq 0\right\}$ be a discrete-time and continuous-state nonhomogeneous Markov chain on the measure $Q$ with the initial distribution density $u(x), x \in R$ and the transition probability density $p_{n}=p_{n}(x, y), x, y \in R, n \geq 1$. Then for any Borel, set $B$

$$
\begin{aligned}
& Q\left(X_{0} \in B\right)=\int_{B} u(x) d x, \\
& Q\left(X_{n+1} \in B \mid X_{n}=x\right)=\int_{B} p_{n}(x, y) d y,
\end{aligned}
$$

then

$$
g_{n}\left(x_{0}, x_{1}, \ldots, x_{n}\right)=u\left(x_{0}\right) p_{0}\left(x_{0}, x_{1}\right) \cdots p_{n-1}\left(x_{n-1}, x_{n}\right)=u\left(x_{0}\right) \prod_{k=1}^{n} p_{k-1}\left(x_{k-1}, x_{k}\right),
$$

so

$$
r_{n}(\omega)=\frac{u\left(X_{0}\right) \prod_{k=1}^{n} p_{k-1}\left(X_{k-1}, X_{k}\right)}{f_{n}\left(X_{0}, X_{1} \cdots X_{n}\right)} .
$$

There have been some works on deviation theorem, a kind of strong limit theorem represented by inequalities. Liu and Yang [3] have studied the limit properties of a class of averages of functions of two variables of arbitrary information sources. Liu and Yang [4] investigated the strong deviation theorems for arbitrary information source relative to Markov information source. Liu [5] discussed a class of strong deviation theorems for an arbitrary stochastic sequence with respect to the marginal distribution by using generating function method, and also studied the problem above by means of Laplace transform [6]. Liu and Wang [7] have studied a strong limit theorem expressed by inequalities for the sequences of absolutely continuous random variables. Recently, Fan [8] has studied some strong deviation theorems for dependent continuous random sequence.

In this paper, by using the notion of asymptotic log-likehood and the martingale convergence theorem, and extending the analytic technique proposed by Liu [9], Liu and Yang [7] to the case of discrete-time and continuous-state nonhomogeneous Markov chains, we obtain the strong deviation theorem for discrete-time and continuous-state nonhomogeneous Markov chains.

\section{Main result}

Theorem 1 Let $\left\{X_{n}, n \geq 0\right\}$ be a discrete-time and continuous-state nonhomogeneous Markov chain on the measure $Q, r(\omega)$ and $r_{n}(\omega)$ be defined by (1) and (2), respectively. Let $\left\{B_{n}, n \geq 1\right\}$ be a sequence of Borel set of the real line, and $I_{B_{n}}$ be the indicative function of $B_{n}$. Let

$$
a(\omega)=\limsup _{n \rightarrow \infty} \frac{1}{n} \sum_{k=1}^{n} \int_{B_{k}} p_{k-1}\left(X_{k-1}, x_{k}\right) d x_{k} \leq b, \quad \forall \omega \in \Omega
$$

and

$$
D_{1}=\{\omega: r(\omega) \leq b\}, \quad D_{2}=\{\omega: r(\omega) \geq b\} .
$$


Then

(a) $\quad \limsup _{n \rightarrow \infty} \frac{1}{n} \sum_{k=1}^{n}\left[I_{B_{k}}\left(X_{k}\right)-\int_{B_{k}} p_{k-1}\left(X_{k-1}, x_{k}\right) d x_{k}\right] \leq 2 \sqrt{b r(\omega)}+r(\omega) \quad$ a.s.;
(b) $\liminf _{n \rightarrow \infty} \frac{1}{n} \sum_{k=1}^{n}\left[I_{B_{k}}\left(X_{k}\right)-\int_{B_{k}} p_{k-1}\left(X_{k-1}, x_{k}\right) d x_{k}\right] \geq-2 \sqrt{b r(\omega)}$ a.s. on $D_{1}$,

and

$$
\liminf _{n \rightarrow \infty} \frac{1}{n} \sum_{k=1}^{n}\left[I_{B_{k}}\left(X_{k}\right)-\int_{B_{k}} p_{k-1}\left(X_{k-1}, x_{k}\right) d x_{k}\right] \geq-b-r(\omega) \quad \text { a.s. on } D_{2} \text {. }
$$

Proof Let $\lambda$ be a nonnegative constant, and let

$$
h_{k}\left(x_{k-1}, x_{k}\right)= \begin{cases}\frac{\lambda p_{k-1}\left(x_{k-1}, x_{k}\right)}{1+(\lambda-1) \int_{B_{k}} p_{k-1}\left(x_{k-1}, x_{k}\right) d x_{k}}, & x_{k} \in B_{k} ; \\ \frac{p_{k-1}\left(x_{k-1}, x_{k}\right)}{1+(\lambda-1) \int_{B_{k}} p_{k-1}\left(x_{k-1}, x_{k}\right) d x_{k}}, & x_{k} \notin B_{k} .\end{cases}
$$

It is easy to see that $u\left(x_{0}\right) \prod_{k=1}^{n} h\left(x_{k-1}, x_{k}\right)$ is a density function of $n+1$ variables. Let

$$
t_{n}(\lambda, \omega)=\frac{u\left(X_{0}\right) \prod_{k=1}^{n} h_{k}\left(X_{k-1}, X_{k}\right)}{f_{n}\left(X_{0}, X_{1} \cdots X_{n}\right)}
$$

then $t_{n}(\lambda, \omega)$ is a nonnegative supermartingale that converges a.s. Hence there exists $A(\lambda) \in \mathcal{F}, P(A(\lambda))=1$ such that

$$
\limsup _{n \rightarrow \infty} \frac{1}{n} \ln t_{n}(\lambda, \omega) \leq 0, \quad \omega \in A(\lambda)
$$

Letting $\lambda=1$ in (9), we obtain

$$
\limsup _{n \rightarrow \infty} \frac{1}{n} \ln r_{n}(\omega) \leq 0, \quad \omega \in A(1) .
$$

This implies that

$$
r(\omega) \geq 0, \quad \omega \in A(1) .
$$

We have by (7)

$$
\begin{aligned}
\prod_{k=1}^{n} h_{k}\left(X_{k-1}, X_{k}\right) & =\prod_{k=1}^{n} \frac{\lambda^{I_{B_{k}}\left(X_{k}\right)} p_{k-1}\left(X_{k-1}, X_{k}\right)}{1+(\lambda-1) \int_{B_{k}} p_{k-1}\left(X_{k-1}, x_{k}\right) d x_{k}} \\
& =\lambda^{\sum_{k=1}^{n} I_{B_{k}}\left(X_{k}\right)} \prod_{k=1}^{n} \frac{p_{k-1}\left(X_{k-1}, X_{k}\right)}{1+(\lambda-1) \int_{B_{k}} p_{k-1}\left(X_{k-1}, x_{k}\right) d x_{k}} .
\end{aligned}
$$

It follows from (2), (8), and (12) that

$$
\ln t_{n}(\lambda, \omega)=\sum_{k=1}^{n} I_{B_{k}}\left(X_{k}\right) \ln \lambda-\sum_{k=1}^{n} \ln \left[1+(\lambda-1) \int_{B_{k}} p_{k-1}\left(X_{k-1}, x_{k}\right) d x_{k}\right]+\ln r_{n}(\omega) .
$$


By (9) and (13), we have

$$
\begin{aligned}
& \limsup _{n \rightarrow \infty} \frac{1}{n}\left(\sum_{k=1}^{n} I_{B_{k}}\left(X_{k}\right) \ln \lambda+\ln r_{n}(\omega)-\sum_{k=1}^{n} \ln \left[1+(\lambda-1) \int_{B_{k}} p_{k-1}\left(X_{k-1}, x_{k}\right) d x_{k}\right]\right) \\
& \leq 0, \quad \omega \in A(\lambda) .
\end{aligned}
$$

(a) Let $\lambda>1$. Dividing the two sides of (14) by $\ln \lambda$, we obtain

$$
\begin{aligned}
& \limsup _{n \rightarrow \infty} \frac{1}{n}\left(\sum_{k=1}^{n} I_{B_{k}}\left(X_{k}\right)+\frac{\ln r_{n}(\omega)}{\ln \lambda}-\sum_{k=1}^{n} \frac{\ln \left[1+(\lambda-1) \int_{B_{k}} p_{k-1}\left(X_{k-1}, x_{k}\right) d x_{k}\right]}{\ln \lambda}\right) \\
& \leq 0, \quad \omega \in A(\lambda) .
\end{aligned}
$$

By (1) and (15), we have

$$
\begin{aligned}
& \limsup _{n \rightarrow \infty} \frac{1}{n}\left(\sum_{k=1}^{n} I_{B_{k}}\left(X_{k}\right)-\sum_{k=1}^{n} \frac{\ln \left[1+(\lambda-1) \int_{B_{k}} p_{k-1}\left(X_{k-1}, x_{k}\right) d x_{k}\right]}{\ln \lambda}\right) \\
& \quad \leq \frac{r(\omega)}{\ln \lambda}, \quad \omega \in A(\lambda) .
\end{aligned}
$$

By (3), (16), the property of the superior limit

$$
\limsup _{n \rightarrow \infty}\left(a_{n}-b_{n}\right) \leq d \Rightarrow \limsup _{n \rightarrow \infty}\left(a_{n}-c_{n}\right) \leq \limsup _{n \rightarrow \infty}\left(b_{n}-c_{n}\right)+d
$$

and the inequality $0 \leq \ln (1+x) \leq x(x \geq 0)$, we have

$$
\begin{aligned}
& \limsup _{n \rightarrow \infty} \frac{1}{n} \sum_{k=1}^{n}\left[I_{B_{k}}\left(X_{k}\right)-\int_{B_{k}} p_{k-1}\left(X_{k-1}, x_{k}\right) d x_{k}\right] \\
& \leq \limsup _{n \rightarrow \infty} \frac{1}{n} \sum_{k=1}^{n}\left[\frac{\ln \left[1+(\lambda-1) \int_{B_{k}} p_{k-1}\left(X_{k-1}, x_{k}\right) d x_{k}\right]}{\ln \lambda}-\int_{B_{k}} p_{k-1}\left(X_{k-1}, x_{k}\right) d x_{k}\right] \\
& \quad+\frac{r(\omega)}{\ln \lambda} \\
& \leq \limsup _{n \rightarrow \infty} \frac{1}{n} \sum_{k=1}^{n}\left[\frac{(\lambda-1) \int_{B_{k}} p_{k-1}\left(X_{k-1}, x_{k}\right) d x_{k}}{\ln \lambda}-\int_{B_{k}} p_{k-1}\left(X_{k-1}, x_{k}\right) d x_{k}\right]+\frac{r(\omega)}{\ln \lambda} \\
& \leq b\left(\frac{\lambda-1}{\ln \lambda}-1\right)+\frac{r(\omega)}{\ln \lambda}, \quad \omega \in A(\lambda) .
\end{aligned}
$$

By using the inequality $1-\frac{1}{\lambda}<\ln \lambda(\lambda>1)$, we have by (17)

$$
\limsup _{n \rightarrow \infty} \frac{1}{n} \sum_{k=1}^{n}\left[I_{B_{k}}\left(X_{k}\right)-\int_{B_{k}} p_{k-1}\left(X_{k-1}, x_{k}\right) d x_{k}\right] \leq b(\lambda-1)+\frac{\lambda r(\omega)}{\lambda-1}, \quad \omega \in A(\lambda) .
$$

Let $Q^{*}$ be the set of rational numbers in the interval $(1,+\infty)$, and let

$$
A^{*}=\bigcap_{\lambda \in Q^{*}} A(\lambda), \quad g(\lambda, r)=b(\lambda-1)+\frac{\lambda r}{\lambda-1} .
$$


Then we have by (18),

$$
\limsup _{n \rightarrow \infty} \frac{1}{n} \sum_{k=1}^{n}\left[I_{B_{k}}\left(X_{k}\right)-\int_{B_{k}} p_{k-1}\left(X_{k-1}, x_{k}\right) d x_{k}\right] \leq g(\lambda, r(\omega)), \quad \omega \in A^{*}, \lambda \in Q^{*}
$$

Let $b>0$. It is easy to see if $r>0, g(\lambda, r)$ as a function of $\lambda$ attains its smallest value $g\left(1+\sqrt{\frac{r}{b}}, r\right)=2 \sqrt{b r}+r$ on the interval $(1,+\infty)$, and $g(\lambda, 0)$ is increasing on the interval $(1,+\infty)$ and $\lim _{\lambda \rightarrow 1+0} g(\lambda, 0)=0$. For each $\omega \in A^{*} \cap A(1)$ if $r(\omega) \neq \infty$, take $\lambda_{n}(\omega) \in Q^{*}, n=$ $1,2, \ldots$ such that $\lambda_{n}(\omega) \rightarrow 1+\sqrt{\frac{r(\omega)}{b}}$, we have

$$
\lim _{n \rightarrow \infty} g\left(\lambda_{n}(\omega), r(\omega)\right)=2 \sqrt{b r(\omega)}+r(\omega)
$$

By (20), we have

$$
\limsup _{n \rightarrow \infty} \frac{1}{n} \sum_{k=1}^{n}\left[I_{B_{k}}\left(X_{k}\right)-\int_{B_{k}} p_{k-1}\left(X_{k-1}, x_{k}\right) d x_{k}\right] \leq g\left(\lambda_{n}(\omega), r(\omega)\right), \quad n=1,2, \ldots
$$

By (21) and (22), we have

$$
\begin{gathered}
\limsup _{n \rightarrow \infty} \frac{1}{n} \sum_{k=1}^{n}\left[I_{B_{k}}\left(X_{k}\right)-\int_{B_{k}} p_{k-1}\left(X_{k-1}, x_{k}\right) d x_{k}\right] \\
\leq 2 \sqrt{b r(\omega)}+r(\omega), \quad \omega \in A^{*} \cap A(1) .
\end{gathered}
$$

If $r(\omega)=\infty$, (23) holds obviously. Since $P\left(A^{*} \cap A(1)\right)=1$, (4) holds by (23) when $b>0$.

When $b=0$, letting $\lambda=e$ in (20), we have

$$
\limsup _{n \rightarrow \infty} \frac{1}{n} \sum_{k=1}^{n}\left[I_{B_{k}}\left(X_{k}\right)-\int_{B_{k}} p_{k-1}\left(X_{k-1}, x_{k}\right) d x_{k}\right] \leq r(\omega), \quad \omega \in A(e) .
$$

Since $P(A(e))=1,(4)$ also holds by (24) when $b=0$.

(b) Let $0<\lambda<1$. Dividing the two sides of (14) by $\ln \lambda$, we have

$$
\begin{aligned}
& \liminf _{n \rightarrow \infty} \frac{1}{n}\left(\sum_{k=1}^{n} I_{B_{k}}\left(X_{k}\right)-\sum_{k=1}^{n} \frac{\ln \left[1+(\lambda-1) \int_{B_{k}} p_{k-1}\left(X_{k-1}, x_{k}\right) d x_{k}\right]}{\ln \lambda}+\frac{\ln r_{n}(\omega)}{\ln \lambda}\right) \\
& \geq 0, \quad \omega \in A(\lambda) .
\end{aligned}
$$

By (1) and (25), we have

$$
\begin{aligned}
& \liminf _{n \rightarrow \infty} \frac{1}{n}\left(\sum_{k=1}^{n} I_{B_{k}}\left(X_{k}\right)-\sum_{k=1}^{n} \frac{\ln \left[1+(\lambda-1) \int_{B_{k}} p_{k-1}\left(X_{k-1}, x_{k}\right) d x_{k}\right]}{\ln \lambda}\right) \\
& \geq \frac{r(\omega)}{\ln \lambda}, \quad \omega \in A(\lambda) .
\end{aligned}
$$

By (26), (3), the property of the inferior limit

$$
\liminf _{n \rightarrow \infty}\left(a_{n}-b_{n}\right) \geq d \Rightarrow \liminf _{n \rightarrow \infty}\left(a_{n}-c_{n}\right) \geq \liminf _{n \rightarrow \infty}\left(b_{n}-c_{n}\right)+d,
$$


and the inequality $\ln (1+x) \leq x(-1<x \leq 0)$, we have

$$
\begin{aligned}
\liminf _{n \rightarrow \infty} & \frac{1}{n} \sum_{k=1}^{n}\left[I_{B_{k}}\left(X_{k}\right)-\int_{B_{k}} p_{k-1}\left(X_{k-1}, x_{k}\right) d x_{k}\right] \\
\geq & \liminf _{n \rightarrow \infty} \frac{1}{n} \sum_{k=1}^{n}\left[\frac{\ln \left[1+(\lambda-1) \int_{B_{k}} p_{k-1}\left(X_{k-1}, x_{k}\right) d x_{k}\right]}{\ln \lambda}-\int_{B_{k}} p_{k-1}\left(X_{k-1}, x_{k}\right) d x_{k}\right] \\
& +\frac{r(\omega)}{\ln \lambda} \\
\geq & \liminf _{n \rightarrow \infty} \frac{1}{n} \sum_{k=1}^{n}\left[\frac{(\lambda-1) \int_{B_{k}} p_{k-1}\left(X_{k-1}, x_{k}\right) d x_{k}}{\ln \lambda}-\int_{B_{k}} p_{k-1}\left(X_{k-1}, x_{k}\right) d x_{k}\right]+\frac{r(\omega)}{\ln \lambda} \\
\geq & b\left(\frac{\lambda-1}{\ln \lambda}-1\right)+\frac{r(\omega)}{\ln \lambda}, \quad \omega \in A(\lambda) .
\end{aligned}
$$

By using the inequality $1-\frac{1}{\lambda}<\ln \lambda<0$ and $\ln \lambda<\lambda-1<0(0<\lambda<1)$, we have by (27)

$$
\begin{gathered}
\liminf _{n \rightarrow \infty} \frac{1}{n} \sum_{k=1}^{n}\left[I_{B_{k}}\left(X_{k}\right)-\int_{B_{k}} p_{k-1}\left(X_{k-1}, x_{k}\right) d x_{k}\right] \\
\geq b(\lambda-1)+\frac{r(\omega)}{\lambda-1}, \quad \omega \in A(\lambda) \cap A(1) .
\end{gathered}
$$

Let $Q$ * be the set of rational numbers in the interval $(0,1)$, and let

$$
A *=\bigcap_{\lambda \in Q^{*}} A(\lambda), \quad h(\lambda, r)=b(\lambda-1)+\frac{r}{\lambda-1} .
$$

Then we have by (28)

$$
\begin{gathered}
\liminf _{n \rightarrow \infty} \frac{1}{n} \sum_{k=1}^{n}\left[I_{B_{k}}\left(X_{k}\right)-\int_{B_{k}} p_{k-1}\left(X_{k-1}, x_{k}\right) d x_{k}\right] \\
\geq h(\lambda, r(\omega)), \quad \omega \in A * \cap A(1), \lambda \in Q^{*} .
\end{gathered}
$$

Let $b>0$. It is easy to see that if $0<r<b$, then $h(\lambda, r)$ as a function of $\lambda$ attains its largest value $h\left(1-\sqrt{\frac{r}{b}}, r\right)=-2 \sqrt{b r}$ on the interval $(0,1)$, and $h(\lambda, 0)$ is increasing on the interval $(0,1)$ and $\lim _{\lambda \rightarrow 1-0} h(\lambda, 0)=0$, and $h(\lambda, b)=b\left(\lambda-1+\frac{1}{\lambda-1}\right)$ is decreasing on the interval $(0,1)$ and $\lim _{\lambda \rightarrow 0^{+}} h(\lambda, b)=-2 b$. For each $\omega \in A_{*} \cap A(1) \cap D_{1}$, take $\tau_{n}(\omega) \in Q *, n=1,2, \ldots$ such that $\tau_{n}(\omega) \rightarrow 1-\sqrt{\frac{r(\omega)}{b}}$. Then we have

$$
\lim _{n \rightarrow \infty} h\left(\tau_{n}(\omega), r(\omega)\right)=-2 \sqrt{b r(\omega)}
$$

By (30), we have

$$
\liminf _{n \rightarrow \infty} \frac{1}{n} \sum_{k=1}^{n}\left[I_{B_{k}}\left(X_{k}\right)-\int_{B_{k}} p_{k-1}\left(X_{k-1}, x_{k}\right) d x_{k}\right] \geq h\left(\tau_{n}(\omega), r(\omega)\right), \quad n=1,2, \ldots
$$


By (31) and (32),

$$
\begin{gathered}
\liminf _{n \rightarrow \infty} \frac{1}{n} \sum_{k=1}^{n}\left[I_{B_{k}}\left(X_{k}\right)-\int_{B_{k}} p_{k-1}\left(X_{k-1}, x_{k}\right) d x_{k}\right] \\
\geq-2 \sqrt{b r(\omega)}, \quad \omega \in A * \cap A(1) \cap D_{1} .
\end{gathered}
$$

Since $P(A * \cap A(1))=1$, (5) holds by (33) when $b>0$.

When $b=0, r(\omega)=0$ for $\omega \in D_{1} \cap A(1)$, hence we have by (30)

$$
\liminf _{n \rightarrow \infty} \frac{1}{n} \sum_{k=1}^{n}\left[I_{B_{k}}\left(X_{k}\right)-\int_{B_{k}} p_{k-1}\left(X_{k-1}, x_{k}\right) d x_{k}\right] \geq 0, \quad \omega \in A(\lambda) \cap A(1) \cap D_{1},
$$

since $P(A(\lambda) \cap A(1))=1$, (5) also holds by (34) when $b=0$.

It is easy to see that when $0 \leq b<r, h(\lambda, r)$ as a function of $\lambda$ is decreasing on the interval $(0,1)$ and $\lim _{\lambda \rightarrow 0^{+}} h(\lambda, r)=-(r+b)$. For each $\omega \in A^{*} \cap A(1) \cap D_{2}$, when $r(\omega) \neq \infty$, take $\lambda_{n}(\omega) \in Q *, n=1,2, \ldots$, such that $\lambda_{n}(\omega) \rightarrow 0$. We have

$$
\lim _{n \rightarrow \infty} h\left(\lambda_{n}(\omega), r(\omega)\right)=-r(\omega)-b
$$

By (30), we have

$$
\liminf _{n \rightarrow \infty} \frac{1}{n} \sum_{k=1}^{n}\left[I_{B_{k}}\left(X_{k}\right)-\int_{B_{k}} p_{k-1}\left(X_{k-1}, x_{k}\right) d x_{k}\right] \geq h\left(\lambda_{n}(\omega), r(\omega)\right), \quad n=1,2, \ldots
$$

It follows from (35) and (36) that

$$
\begin{gathered}
\liminf _{n \rightarrow \infty} \frac{1}{n} \sum_{k=1}^{n}\left[I_{B_{k}}\left(X_{k}\right)-\int_{B_{k}} p_{k-1}\left(X_{k-1}, x_{k}\right) d x_{k}\right] \\
\geq-r(\omega)-b, \quad \omega \in A * \cap A(1) \cap D_{2}
\end{gathered}
$$

when $r(\omega)=\infty$, (37) also holds obviously. Since $P(A * \cap A(1))=1$, (6) follows from (37) directly.

\section{Competing interests}

The authors declare that they have no competing interests.

\section{Authors' contributions}

BW carried out the study of strong deviation theorem and drafted the manuscript. ZS participated in the proof of theorem. All authors read and approved the final manuscript.

\section{Acknowledgements}

This work is supported by the National Natural Science Foundations of China (11071104, 11226210), and the Research Foundation for Advanced Talents of Jiangsu University (11JDG116).

Received: 22 May 2013 Accepted: 9 September 2013 Published: 07 Nov 2013

\section{References}

1. Liu, W: Strong Deviation and Analytic Method. Science Press, Beijing (2003)

2. Wang, B: Strong law of large numbers for discrete-time and continuous-state nonhomogeneous Markov chains. J. Jiangsu Univ. Nat. Sci. 29(1), 86-88 (2008) 
3. Liu, W, Yang, WG: The limit properties of a class of averages of functions of two variables of arbitrary information sources. Chinese J. Appl. Probab. Statist. 11(2), 195-203 (1995)

4. Liu, W, Yang, WG: A comparison between arbitrary information sources and nonhomogeneous Markov information sources and some small deviations theorems. Acta Math. Sin. (Chin. Ser.) 40(1), 22-36 (1997)

5. Liu, W, Yang, WG: The Markov approximation of the sequences of $N$-valued random variables and a class of small deviation theorems. Stoch. Process. Appl. 89, 117-130 (2000)

6. Liu, W: A class of strong deviation theorems and Laplace transform methods. Chin. Sci. Bull. 43(10), 1036-1041 (1998)

7. Liu, W, Wang, YJ: A strong limit theorem expressed by inequalities for the sequences of absolutely continuous random variables. Hiroshima Math. J. 32, 379-387 (2002)

8. Fan, AH: Some strong deviation theorems for dependent continuous random sequence. Chin. Q. J. Math. 25(4), 572-577 (2010)

9. Liu, W: Relative entropy densities and a class of limit theorems of the sequences of $m$-valued random variables. Ann. Probab. 18, 829-839 (1990)

10.1186/1029-242X-2013-462

Cite this article as: Wang and Shi: The strong deviation theorem for discrete-time and continuous-state

nonhomogeneous Markov chains. Journal of Inequalities and Applications 2013, 2013:462

\section{Submit your manuscript to a SpringerOpen ${ }^{\circ}$ journal and benefit from:}

- Convenient online submission

- Rigorous peer review

- Immediate publication on acceptance

- Open access: articles freely available online

- High visibility within the field

- Retaining the copyright to your article 